\title{
Cell-type-specific regulation of genes involved in testicular lipid metabolism: fatty acid-binding proteins, diacylglycerol acyltransferases, and perilipin 2
}

\author{
Gerardo M Oresti*, Jesús García-López ${ }^{1, *}$, Marta I Aveldaño and Jesús del Mazo ${ }^{1}$ \\ Instituto de Investigaciones Bioquímicas de Bahía Blanca (INIBIBB), Centro Científico Tecnológico Bahía Blanca, \\ Consejo Nacional de Investigaciones Científicas y Técnicas (CONICET), Universidad Nacional del Sur (UNS), \\ CC 857, 8000 Bahía Blanca, Argentina and ${ }^{7}$ Department of Cellular and Molecular Biology, Centro de Investigaciones \\ Biológicas, Consejo Superior de Investigaciones Científicas (CSIC), Ramiro de Maeztu 9, 28040 Madrid, Spain
}

Correspondence should be addressed to G M Oresti; Email: gmoresti@criba.edu.ar

*G M Oresti and J García-López contributed equally to this work

\begin{abstract}
Male germ cell differentiation entails the synthesis and remodeling of membrane polar lipids and the formation of triacylglycerols (TAGs). This requires fatty acid-binding proteins (FABPs) for intracellular fatty acid traffic, a diacylglycerol acyltransferase (DGAT) to catalyze the final step of TAG biosynthesis, and a TAG storage mode. We examined the expression of genes encoding five members of the FABP family and two DGAT proteins, as well as the lipid droplet protein perilipin 2 (PLIN2), during mouse testis development and in specific cells from seminiferous epithelium. Fabp5 expression was distinctive of Sertoli cells and consequently was higher in prepubertal than in adult testis. The expression of Fabp3 increased in testis during postnatal development, associated with the functional differentiation of interstitial cells, but was low in germ cells. Fabp9, together with Fabp12, was prominently expressed in the latter. Their transcripts increased from spermatocytes to spermatids and, interestingly, were highest in spermatid-derived residual bodies (RB). Both Sertoli and germ cells, which produce neutral lipids and store them in lipid droplets, expressed Plin2. Yet, while Dgat1 was detected in Sertoli cells, Dgat2 accumulated in germ cells with a similar pattern of expression as Fabp9. These results correlated with polyunsaturated fatty acid-rich TAG levels also increasing with mouse germ cell differentiation highest in RB, connecting DGAT2 with the biosynthesis of such TAGs. The age- and germ cell type-associated increases in Fabp9, Dgat2, and Plin2 levels are thus functionally related in the last stages of germ cell differentiation.
\end{abstract}

Reproduction (2013) 146 471-480

\section{Introduction}

During spermatogenesis, the extensive formation and remodeling of membranes associated with germ cell differentiation and maturation entail considerable quantitative and qualitative changes in their lipids. In rodents, classical studies have shown that testicular glycerophospholipids (GPLs) and triacylglycerols (TAGs) become increasingly rich in long-chain polyunsaturated fatty acids (PUFAs) like docosapentaenoic acid during postnatal development, as spermatids contain more of this fatty acid in their GPLs and TAGs than spermatocytes (Beckman et al. 1978, Grogan \& Huth 1983). Concomitantly, there are also significant changes in unique fatty acids of testicular sphingomyelins and ceramides destined to be components of spermatozoa (Zanetti et al. 2010): the proportion of 2-hydroxy very-long-chain PUFA with respect to their nonhydroxy counterparts increases considerably in both lipids from spermatocytes and spermatids (Oresti et al. 2010). The regulation of fatty acid biosynthesis and exchange among intracellular organelles of germ cells during spermatogenesis are thus essential for male fertility.

In the final stages of spermatogenesis, spermatids elongate and undergo a considerable cytoplasmic volume reduction via the formation and release of the membrane-bound vesicles known as 'residual bodies' (RB). These densely packed structures, readily phagocytized by Sertoli cells (Kerr \& De Kretser 1974), contain remains of cytoplasm, RNA, former organelles, and neutral lipid inclusions (Russell et al. 1990). The spermatid-derived RB are clearly discernible by markers of neutral lipid droplets, in accordance with the finding that they are even richer in TAGs than spermatids (Oresti et al. 2010).

The interstitium-located Leydig cells are involved in the production of testosterone. The regulatory functions performed by Sertoli cells require a continuous cross 
talk between them and the germ cells they nurse in the seminiferous epithelium. Both these functions are under autocrine, paracrine, and endocrine regulation. The interdependent cellular structure and functions of the testis thus provide a paradigmatic model to assess the regulation and relevance of the different genes encoding for proteins involved in lipid biosynthesis and catabolism, which require fatty acid traffic among membranes throughout development and cell differentiation.

Fatty acid-binding proteins (FABPs) comprise a conserved family of small cytosolic proteins that reversibly bind long-chain fatty acids and their corresponding acyl-CoA esters. They promote the uptake and intracellular transport of fatty acids, guide them to specific metabolic pathways, and participate in cell growth by regulating the expression of specific genes (Haunerland \& Spener 2004, Furuhashi \& Hotamisligil 2008). In mammals, ten FABP members with different tissue-dependent patterns of expression have been described (Yamamoto et al. 2009). All FABPs have the same primary role with potential differences in ligand selectivity for long-chain fatty acids, binding affinity, and binding mechanism. The expression of FABPs in each particular cell type has been considered to reflect its lipid-metabolizing capacity (Chmurzynska 2006).

The predominant FABP in testis is widely accepted to be FABP9 (Kido \& Namiki 2000, Selvaraj et al. 2010), although other members of this family have also been reported, such as FABP3 (Watanabe et al. 1991), FABP5 (Kingma et al. 1998), and more recently FABP12 (Liu et al. 2008, Yamamoto et al. 2009). However, no studies are available comparing on the same basis the expression of different FABPs at definite periods of testis development and correlating this with their relative abundances, in particular testicular cell types.

The biosynthesis of TAG involves several enzymatic steps. The last of these, in which a long-chain fatty acylCoA is joined to a diacylglycerol molecule, is catalyzed by the enzyme acyl-CoA:diacylglycerol acyltransferase (DGAT), with ubiquitous activity in cells of most tissues (Lehner \& Kuksis 1996). Two DGAT proteins have been described, DGAT1 and DGAT2, which probably differ in physiological function (Cases et al. 1998, 2001). To date, the expression of genes encoding for each of these proteins in testis as a function of developmental stages and in differentiating spermatogenic cells has not yet been reported.

Perilipin 2 (PLIN2), formerly termed adipose differentiation-related protein (ADRP) (Kimmel et al. 2010), is one of the proteins involved in maintaining lipid droplet structure and function (Mclntosh et al. 2012). In the testis, the protein was found to be present in Sertoli and Leydig cells, associated with TAG- and cholesterol estercontaining lipid droplets (Brasaemle et al. 1997, Heid et al. 1998). The abundance of lipid droplets containing TAG - but no cholesterol esters - in rat germ cells and RB (Oresti et al. 2010) suggested the possibility that, if a similar accumulation of TAGs and lipid droplets occurs in mice as germ cell differentiation proceeds, the expression of one of the Dgat genes and Plin2 should coincide with increasing germ cell maturation.

In order to assess the co-regulation of genes involved in lipid metabolism in testis, in this study we analyzed the expression of genes encoding for five FABPs, two DGATs, and PLIN2 in mouse testis during postnatal development and in specific testicular cell types. The inclusion of RB in the germ cell analysis led to the biologically relevant finding that some of these mRNAs are highly concentrated in these particles, whose contents are destined to be recycled by Sertoli cells.

\section{Materials and methods}

\section{Animals}

CD-1 mice were bred at the CIB-CSIC animal facility under specific pathogen-free, temperature $\left(22 \pm 1{ }^{\circ} \mathrm{C}\right)$, and humidity-controlled (50-55\%) conditions, on $12 \mathrm{~h}$ light:12 h darkness cycles and with ad libitum access to food and water. Animals were treated according to the guidelines of the CSIC Bioethics Committee.

\section{Tissue and cell isolation}

Whole testes were obtained from mice at postnatal days 6 (P6), P10, and P18 and from adult mice. Pachytene spermatocytes (PS), round spermatids (RS), elongating spermatids (ES), and $\mathrm{RB}$ were obtained from adult mouse testes and enriched using BSA density gradients on STA-PUT (Romrell et al. 1976, Bellve et al. 1977, Paz et al. 2006, Oresti et al. 2010). Sertoli cells were obtained from testes of mice aged 14-18 postnatal days and enriched by primary culture. The cells were maintained for 2 weeks at $37{ }^{\circ} \mathrm{C}$ in a $5 \% \mathrm{CO}_{2} / 95 \%$ air atmosphere and cultured in DMEM:Ham-F12 medium (Gibco, BRL; 1:1), following standard procedures (Karl \& Griswold 1990). The purity of isolated and cultured cells was 90-95\%, as determined by morphological criteria (Supplementary Figure 1, see section on supplementary data given at the end of this article) and RT-PCR using cell-type-specific primers as described previously (Gonzalez-Gonzalez et al. 2008).

\section{Total RNA extraction and analysis of mRNAs by RT-quantitative PCR}

RNA from testes and germ cells was isolated using TRIzol Reagent (Invitrogen) according to the manufacturer's instructions. RNA was resuspended in RNase-free water and its concentration was assessed from the A260:A280 absorbance ratio in a Spectrophotometer ND-1000 (NanoDrop). Samples were stored at $-80{ }^{\circ} \mathrm{C}$ until use. Aliquots containing $0.2 \mu \mathrm{g}$ total RNA were employed to synthesize cDNA in reactions containing $2.5 \mu \mathrm{M}$ Oligo dT17, $1 \times$ First-Strand Buffer (Invitrogen), $0.01 \mathrm{M}$ dithiothreitol, 2 UI RNase inhibitor (RNAsin Promega), $0.5 \mathrm{mM}$ of each dNTP, and 
Table 1 Sequences of primers for RT-qPCR.

\begin{tabular}{|c|c|c|}
\hline Genes & Primer sequences & Amplicon lengths (nt) \\
\hline Fabp3 & $\begin{array}{l}\text { F: atccatgtgcagaagtggaa } \\
\text { R: cactgccatgagtgagagtca }\end{array}$ & 91 \\
\hline Fabp5 & $\begin{array}{l}\text { F: acggctttgaggagtacatga } \\
\text { R: ctcggttttgaccgtgatg }\end{array}$ & 122 \\
\hline Fabp7 & $\begin{array}{l}\text { F: caagaacacagagatcaatttcca } \\
\text { R: catccaaccgaaccacaga }\end{array}$ & 92 \\
\hline Fabp9 & $\begin{array}{l}\text { F: cactgcagacaaccgaaaag } \\
\text { R: tctgtttgccaagccatttt }\end{array}$ & 86 \\
\hline Fabp12 & $\begin{array}{l}\text { F: ttgaaaactacatgaaggaattgg } \\
\text { R: agtgggctttgccagaca }\end{array}$ & 71 \\
\hline Dgat1 & $\begin{array}{l}\text { F: ggtcaaggccaaagctgtc } \\
\text { R: gatctcggtaggtcaggttgtc }\end{array}$ & 95 \\
\hline Dgat2 & $\begin{array}{l}\text { F: tactccaagcccatcaccac } \\
\text { R: ggcatggtacaggtcgatgt }\end{array}$ & 93 \\
\hline Plin2 & $\begin{array}{l}\text { F: cagccaacgtccgagatt } \\
\text { R: actgtgctggctacagaatcc }\end{array}$ & 107 \\
\hline
\end{tabular}

200 U superscript II (Invitrogen). The reactants were taken to a final volume of $20 \mu \mathrm{l}$ with RNase-free water.

The cDNAs resulting from RT were amplified by real-time quantitative PCR (qPCR). Gene expression levels were determined using the CFX96 Touch Real-Time PCR Detection System (Bio-Rad). RT-qPCR was performed in a final volume of $10 \mu \mathrm{l}$ using iQ SYBR Green SuperMix (Bio-Rad) and $0.25 \mu \mathrm{M}$ concentration of each primer. Primer combinations for the specific amplification of the analyzed genes were designed in 'Universal ProbeLibrary' (http://www.rocheapplied-science.com/sis/rtpcr/upl/ezhome.html) and purchased from Roche. The primers used in PCR are listed in Table 1. The RT-PCR products were assessed by $3 \%$ agarose gel electrophoresis. The efficiency of each primer pair was calculated for comparative and normalizing purposes.

The PCR conditions were as follows: 40 cycles of denaturation at $95{ }^{\circ} \mathrm{C}$ for $15 \mathrm{~s}$, annealing and extension at $60^{\circ} \mathrm{C}$ for $1 \mathrm{~min}$, and a final extension step at $72{ }^{\circ} \mathrm{C}$ for $1 \mathrm{~min}$. The PCR products were also examined by melting curve analysis and agarose gel electrophoresis.

\section{Data analysis}

A total of 12 measurements were done for each gene studied at each stage or cell type: three biological replicates, from which four technical replicates were analyzed. Data were normalized according to the previously described $2^{-\Delta \Delta C t}$ method (Livak \& Schmittgen 2001), using peptidylprolyl isomerase A (Ppia) and $\mathrm{H} 2 \mathrm{~A}$ histone family member $\mathrm{Z}(\mathrm{H} 2 \mathrm{afz})$ as reference genes. The relative level of each gene is expressed as the relative change in gene expression (Livak \& Schmittgen 2001).

\section{Antibodies and western blot}

For FABP9 protein expression analysis, a validated anti-FABP9 antibody (Kido et al. 2005) kindly donated by Dr T Kido (University of California San Francisco, CA, USA) was used. Anti-FABP12 (M-12): sc-241412 and anti-actin (I-19): sc-1616 (both from Santa Cruz Biotechnology) were used as primary antibodies. Tissue and cells were lysed and thoroughly homogenized in Tris- $\mathrm{HCl}(\mathrm{pH} 6.8), 5 \mathrm{mM}$ EDTA, 3\% SDS, and $1 \%$ of protease inhibitor cocktail (Roche Applied Science).
Proteins were mixed with denaturing Laemmli buffer, heated to $95{ }^{\circ} \mathrm{C}$ for $5 \mathrm{~min}$, resolved by SDS-PAGE, and subjected to western blotting using the primary antibody, followed by incubation with HRP-conjugated secondary antibody and detection with SuperSignal Chemiluminescent Substrate (Pierce Biotechnology Rockford, IL, USA). The intensity of bands was compared using the Imagej Software (National Institutes of Health, Bethesda, MD, USA), and data were expressed as arbitrary units relative to actin expression.

\section{Nile Red staining and confocal microscopy}

To detect neutral lipid-containing lipid droplets, testes were fixed at $4 \%$ in paraformaldehyde at $4{ }^{\circ} \mathrm{C}$, rinsed overnight in PBS containing $30 \%$ sucrose at $4{ }^{\circ} \mathrm{C}$ for cryoprotection, placed in a small amount of OCT compound Tissue Tek (Sakura Finetek, Torrance, CA, USA), and stored at $-80^{\circ} \mathrm{C}$. Ten micrometer-thick frozen sections were prepared with a cryostat and picked up on poly-L-lysine-coated glass coverslips (Sigma). Then, tissue sections were incubated in PBS to remove OCT and post-fixed in $4 \%$ paraformaldehyde in PBS for $5 \mathrm{~min}$, followed by incubation for $15 \mathrm{~min}$ at room temperature with $1.5 \mu \mathrm{g} / \mathrm{ml}$ Nile Red (Molecular Probes) in PBS. Samples were mounted with Vectashield Mounting Medium with DAPI (Vector Laboratories). All images were captured using a Leica TCS-SP5-AOBS confocal microscope with $40 \times$ oil immersion objectives.

\section{GPL and TAG separation and fatty acid analysis}

After collecting PS, RS, and RB by centrifugation, lipid extracts were prepared and partitioned (Bligh \& Dyer 1959). After phase separation, the organic solvents were evaporated under a nitrogen stream and the samples dissolved in chloroformmethanol $(2: 1 \mathrm{v} / \mathrm{v})$. Aliquots were taken for total lipid phosphorus determination.

Lipid extracts were spotted on thin layer chromatography (TLC) plates under a $\mathrm{N}_{2}$ atmosphere along with commercial standards (Sigma). The neutral lipids were resolved with $n$-hexane:diethyl ether $(80: 20 \mathrm{v} / \mathrm{v})$ to obtain the TAG, and the total polar lipid fraction (mostly GPLs) was recovered from the origin of these plates. The amount of GPL and TAG in germ cells and RB was estimated from their fatty acids. These were measured by gas chromatography (GC) after conversion of the eluted, dried lipids into fatty acid methyl esters. Before GC, the latter were routinely purified by TLC on (pre-washed) silica gel G plates, using hexane:ether $(95: 5 \mathrm{v} / \mathrm{v})$ as solvent. Fatty acid analysis was performed using the conditions and instrumentation described in previous work (Oresti et al. 2010).

\section{Statistical analysis}

The bars shown in figure histograms represent mean values \pm s.D. One-way ANOVA was used to determine the significance of differences between mean values, which were compared using the Bonferroni's test for multiple comparison. In both analyses, the minimum acceptable level of significance was $P<0.05$. Statistics marks (letters) shown in figures compare the different expression rates of a particular gene at different postnatal days or in different cells. Statistical data comparing 


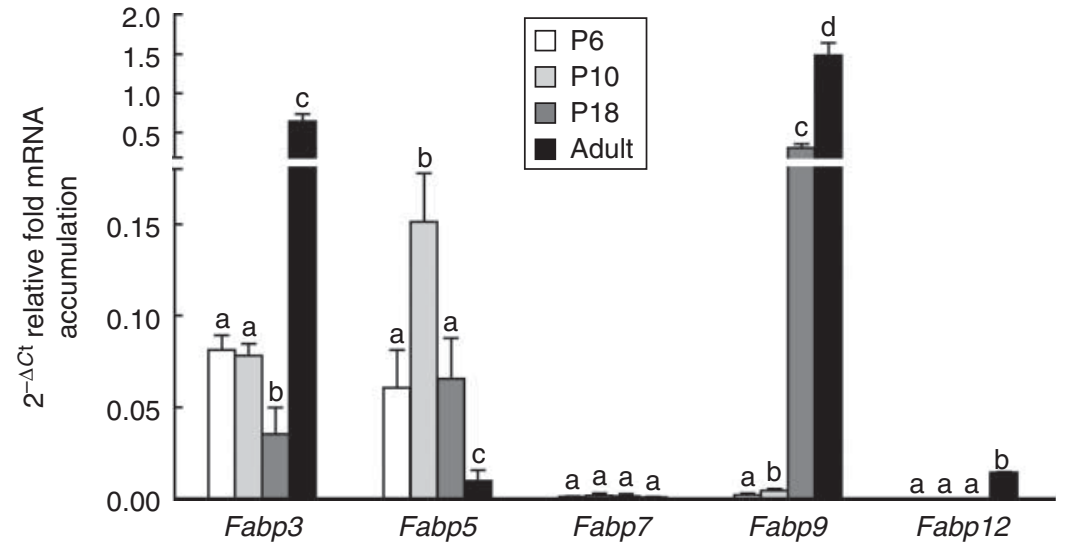

Figure 1 RT-qPCR analyses of Fabp3, Fabp5, Fabp7, Fabp9, and Fabp12 transcripts during postnatal testicular development in the mouse (P6, P10, and P18 refer to postnatal days). Data were normalized to Ppia and $H 2 a f z$ as internal reference using the $2^{-\Delta C}$ method (described in the Materials and methods section). Values with different letters ( $a, b, c$ and d) are significantly different $(P<0.05)$. Statistical significance between different expression rates of all genes studied in a particular postnatal day is shown as supplemental data. the expression rates of the genes studied in a particular postnatal day or between different cells are provided in Supplementary Table 1, see section on supplementary data given at the end of this article.

\section{Results}

\section{Differential expression of FABPS}

Because germ cells at progressively advanced stages of differentiation appear at defined times after birth (Bellve et al. 1977), it was possible to follow the appearance or accumulation of the transcripts under study at specific stages of spermatogenesis. In mice at P6, nearly $85 \%$ of the cells in the seminiferous epithelium correspond to Sertoli cells and $15 \%$ to primitive spermatogonia. At P10, Sertoli cells represent around $50 \%$ of the total cell population, with preleptotene and leptotene spermatocytes, together, accounting for nearly $30 \%$. PS abound at P18, and ES prevail in the adult testis, where they represent about $60 \%$ of the intra-tubular cells, accompanied by an abundant presence of RB (Bellve et al. 1977). The pattern of expression of the five Fabp genes we studied differed markedly during spermatogenic development and cell differentiation (Fig. 1). Analysis by RT-qPCR revealed a relative high transcript accumulation of Fabp3 and Fabp5 during early stages of development (P6-P10), decreasing significantly at P18. Interestingly, from P18 to adult testes, Fabp5 continued to fall whereas Fabp3 showed a significant increase. The expression of Fabp 7 was almost residual in testis and cells (Fig. 1). The Fabp isoform that presented the highest rate of transcript accumulation in adult testis was Fabp9 (1300-fold from P6 to adult). Fabp12 mRNA remained virtually undetectable from P6 to P18 but was clearly more abundant in adult testis (Fig. 1).

Normalized data showed that mRNA levels of Fabp3 were over 20-fold higher in whole adult testis than in any of the seminiferous tubule cell types studied (Fig. 2, left panel). These results strongly suggest that Fabp3 was mainly expressed in extra-tubular cells. Cytological analysis of FABP3 protein during testis development confirmed this assumption, showing its main localization in Leydig cells (Supplementary Figure 2, see section on supplementary data given at the end of this article).

To assess cell-type-specific gene expression in the seminiferous epithelium, comparative analyses were performed in purified Sertoli cells, PS, RS, and RB (Fig. 2). Fabp5 expression was apparently restricted exclusively to Sertoli cells. Because the proportion of Sertoli cells relatively decreases with respect to the massive increase in the number of germ cells from prepubertal to adult

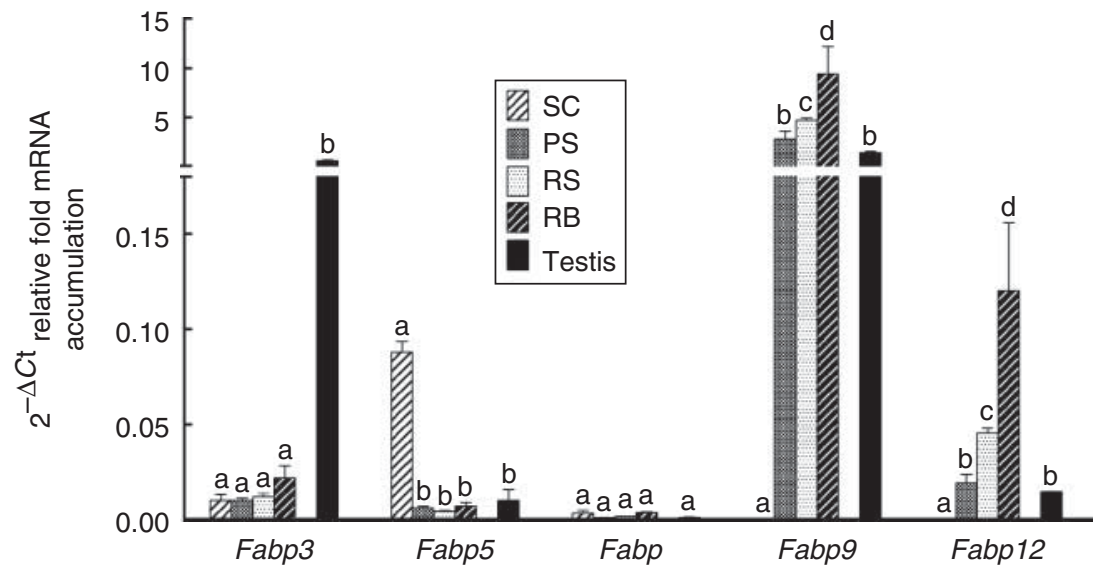

Figure 2 RT-qPCR analyses of Fabp3, Fabp5, Fabp7, Fabp9, and Fabp12 transcripts in different cell types purified from mouse seminiferous tubules. Data were normalized to Ppia and $\mathrm{H} 2 \mathrm{afz}$ as internal reference using the $2^{-\Delta C t}$ method. Values with different letters $(\mathrm{a}, \mathrm{b}, \mathrm{c}$ and $\mathrm{d})$ are significantly different $(P<0.05)$. Statistical significance between different expression rates of all genes studied in a particular cell type is shown as Supplementary Table 1. SC, Sertoli cells. 
testis, this Fabp5 localization explains the apparent progressive reduction observed for its transcript in testis with gonadal development (Fig. 1).

In clear contrast with Fabp5, both Fabp9 and Fabp12 transcript levels were low, virtually absent from Sertoli cells. Their expression was restricted to germ cells and RB. Transcript levels of Fabp9 and Fabp12 increased from spermatocytes to spermatids and reached their maximum values in RB (Fig. 2). In agreement with the lack of expression detected in developing testes, Fabp7 transcripts were virtually absent in the different cell types analyzed.

In view of the predominance of Fabp9 followed by Fabp12 in the germ cell line, we assessed their expression at the protein level at different developmental stages and from isolated cell types (Fig. 3). In western blots, FABP9 was detected as a single band of $15 \mathrm{kDa}$, in agreement with previous work in adult mice (Selvaraj et al. 2010). The protein was undetectable at P6, first appearing at around P18, i.e. associated with the emergence of PS in the first wave of germ cell differentiation. As did its mRNA transcript, FABP9 reached its highest amount in adult testes (Fig. 3A). Also in coincidence with its mRNA distribution, FABP9 protein level was very low in Sertoli cells, considerably higher in germ cells, and as copious in the latest (elongating) spermatids as in RB (Fig. 3A).

FABP12 was detected as two bands of 15 and $17 \mathrm{kDa}$. This may be ascribed in part to post-translational modifications or, as previously suggested for other FABPs (Ockner et al. 1982, Spener et al. 1990), to
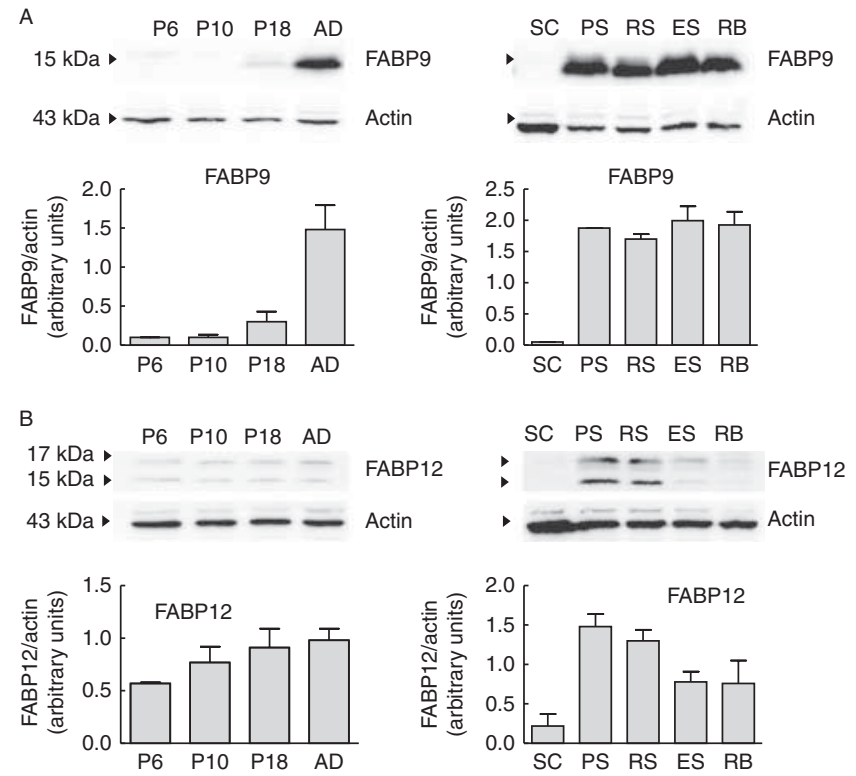

Figure 3 FABP9 (A) and FABP12 (B) protein expression in mouse testis. Western blots from samples obtained from testis during postnatal development and in different cell types obtained from adult testes. AD, adults; SC, Sertoli cells. alternate forms due to this protein binding different amounts of fatty acids or other substrates. FABP12 increased in the testis with postnatal maturation, its level being higher at earlier than at later stages of development (Fig. 3B). This tendency correlated with the distribution of FABP12 among cells: its level was lowest in Sertoli cells and highest in PS and RS but lower than FABP9 in ES and RB (Fig. 3B). This finding points to an interesting difference between these two FABPs in relative rates of mRNA and protein expression during germ cell differentiation.

\section{Dgat and Plin2 expression, neutral lipid droplets, and TAG levels}

The expression profiles shown by Dgat1 and Dgat2 indicated that both genes were subjected to different regulatory mechanisms (Fig. 4A). Although both isoforms were abundantly expressed in the testis at all developmental stages, Dgat2 was the predominant one in the adult. Dgat2 mRNA maintained a low level between P6 and P18 but increased 30-fold from P18 to adult (Fig. 4A). This suggested a preferential association of Dgat2 with spermatids, which was confirmed by the profiles of Dgat1 and Dgat2 expression among cell types (Fig. 4B). Sertoli cells had a low but detectable Dgat 1 while virtually lacked Dgat2 expression. Conversely, in germ cells, Dgat1 mRNA levels were lower, and increased less, than those of Dgat2 with their differentiation. In comparison with spermatocytes, the amount of Dgat2 was three- and fourfold higher in RS and $\mathrm{RB}$ respectively (Fig. 4B).

PLIN2 is one of the proteins associated with neutral lipid-storing intracellular lipid droplets (Brasaemle et al. 1997). Its testicular mRNA levels underwent a total increase of threefold from P6 to adult mouse testes (Fig. 4A), in agreement with the finding that Plin2 was expressed in both Sertoli and germ cells (Fig. 4B). In the latter and RB, its transcript levels were twice as large (Fig. 4B) as in whole testes (Fig. 4A).

In rat testes, the intensity of lipid droplet staining with Nile Red increases from isolated PS to RS and from these cells to RB (Oresti et al. 2010). This allowed linking of this feature with the increase in TAG concentration per cell or particle, which followed the same order. Something similar occurred in the mouse testis, although with qualitative and quantitative differences. Three populations of lipid droplets, one extra-tubular and two within seminiferous tubules, were identified (Fig. 5). The interstitially located ones were considerably larger and more abundant in mice than in rats. Of the intra-tubular droplets, those larger, basally located ones were mostly those included in Sertoli cells, and the smallest ones, facing the tubule lumen, were even smaller and more numerous in mice (Fig. 5) than in rats (Oresti et al. 2010).

The extra-tubular lipid droplets are expected to contain mostly the cholesteryl esters (Brasaemle et al. 1997) that 


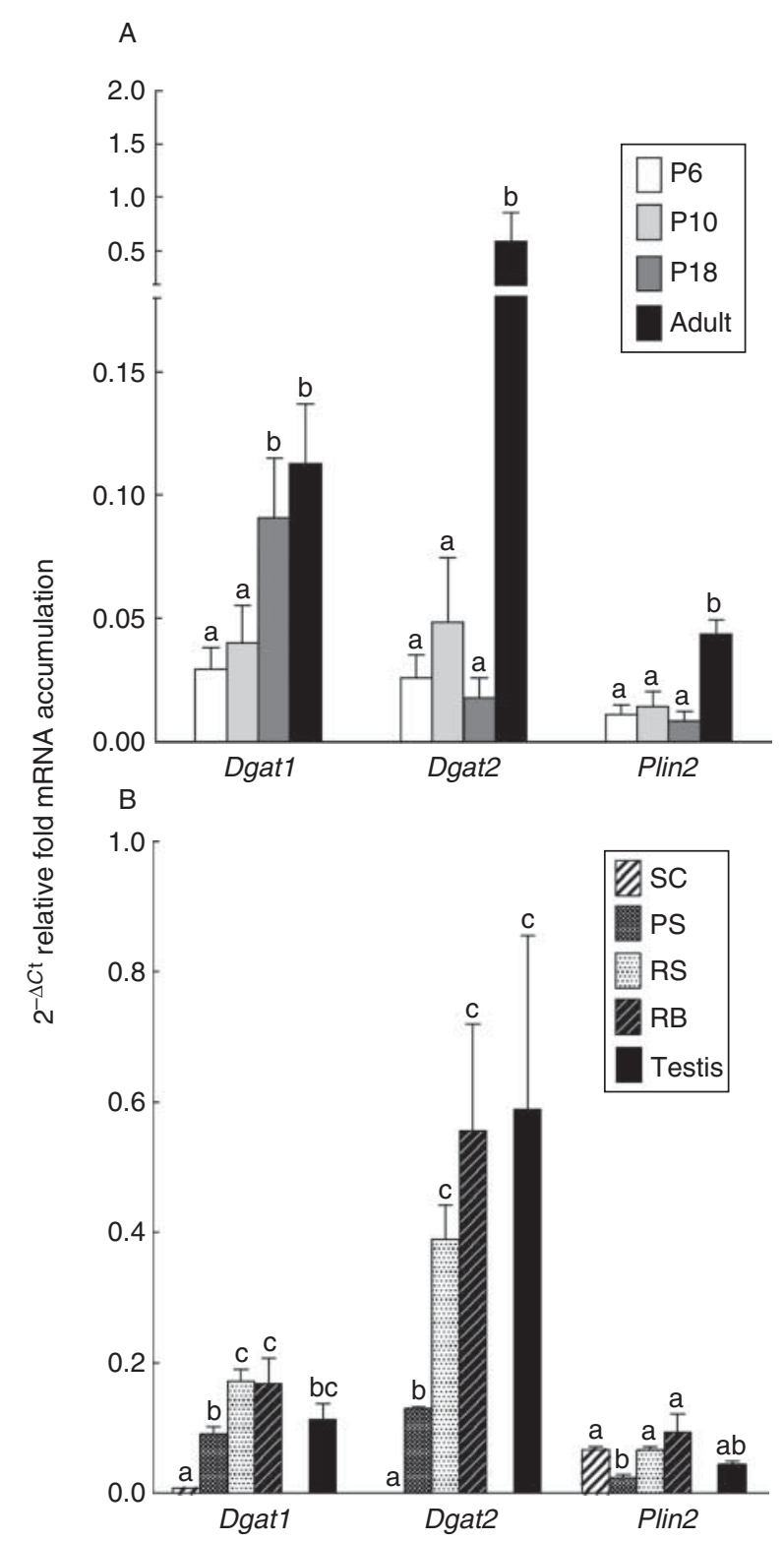

Figure 4 RT-qPCR analyses of Dgat1, Dgat2, and Plin2 transcripts during postnatal development in the mouse testis (A) and in cells from the seminiferous epithelium of adult mice (B). Data were normalized to Ppia and $H 2 a f z$ as internal reference using the $2^{-\Delta C t}$ method. Values with different letters are significantly different $(P<0.05)$. Statistical significance between different expression rates of all genes studied in a particular postnatal day or cell type is shown in Supplementary Table 1. SC, Sertoli cells.

Leydig cells store as a source of the cholesterol involved in steroidogenesis; those of Sertoli cells are probably mixed in nature, as these cells produce both cholesterol esters and TAGs, and those of germ cells mostly contain TAG as the main neutral lipid (Oresti et al. 2010). In this study, we also analyzed the total content (Fig. 5), and that of the main fatty acids (Fig. 6), of the TAG of mouse germ cells and RB, as a manifestation of the TAG-biosynthesizing activity. Concomitantly with the cell location of lipid droplet staining, the expression of Fabp9, Dgat2, and Plin2 correlated with the levels of TAG (Fig. 5) in that all of them increased from PS to RS and from these to RB.

The amounts of main fatty acids in GPLs and TAGs, presented together for comparison in Fig. 6, showed that both lipids from mouse spermatids were significantly richer than those from spermatocytes in two 22-carbon PUFAs, namely docosapentaenoic and docosahexaenoic acids (22:5n- 6 and 22:6n-3). Our results extend previous data by showing that large amounts of these two fatty acids are retained in the testis as spermatozoa are released, as both are significantly increased in the GPL and TAG that accumulate in RB (Fig. 6).

\section{Discussion}

FABPs are ubiquitously expressed proteins with the various members of this family differing in tissue expression patterns (Haunerland \& Spener 2004, Yamamoto et al. 2009). The present quantitative study in the mouse testicular tissue showed, to our knowledge for the first time, cell-compartmentalized and timedependent variations in the gene expression of five simultaneously assessed FABP members, which is indicative of a differential regulation of their expression at specific stages of spermatogenesis.

Our finding that Fabp3 increased eightfold in the testis from the first spermatogenic wave to adult is in agreement with the increase in FABP3 previously reported in a proteomics study (Paz et al. 2006). The developmental profile of Fabp3 expression was consistent with this protein mostly localizing in Leydig cells (Heuckeroth et al. 1987, Watanabe et al. 1991, Zschiesche et al. 1995) and with the fact that intratesticular testosterone concentrations ( $\mathrm{O}^{\prime}$ Shaughnessy et al. 2002), as did Fabp3 (present results), showed a decline between P10 and P20, followed by an increase at P25. We confirmed that FABP3 protein was interstitially located and that it displayed low signal level at P6, decreased at P18, and was plentiful in adult testis (see Fig. 2 in Supplementary Material, see section on supplementary data given at the end of this article). Interestingly, the Fabp3 gene is downregulated in Leydig cells as a result of exposure to mono-2-ethylhexylphthalate (Chauvigne et al. 2011), an environmental endocrine disruptor.

A trait of the protein FABP3 is its higher affinity for arachidonic acid (AA) than for palmitic acid (Murphy et al. 2005). AA is the fatty acid specifically involved in the mechanism of cholesterol transport from the outer to the inner mitochondrial membrane in Leydig cells, the first committed step in steroidogenesis (Albert et al. 1980, Castillo et al. 2006). Taken together, these observations allow the speculation that FABP3 could play a role in testosterone production by transporting the required $\mathrm{AA}$ from other cellular sites to mitochondria. Although there are studies using Fabp3 knockdown mice 

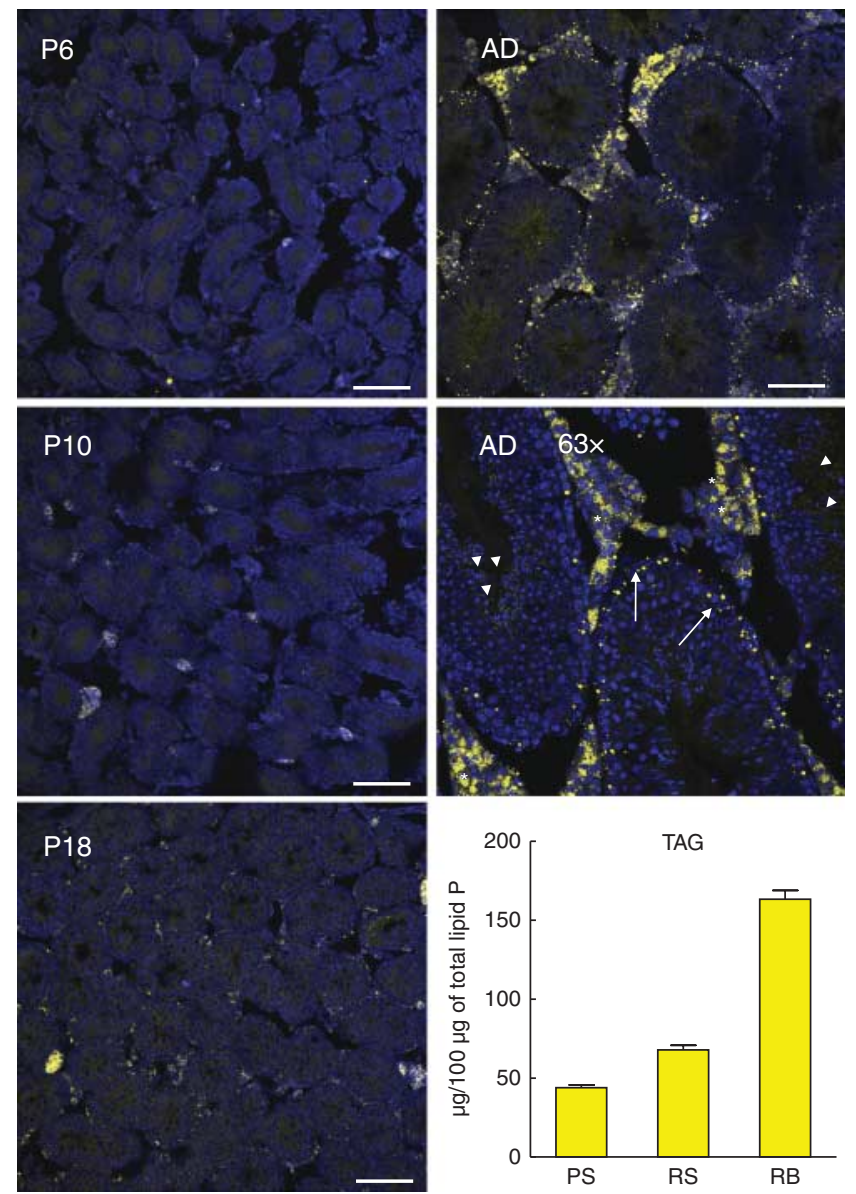

Figure 5 Testicular lipid droplets during postnatal testis development as revealed by Nile Red staining and fluorescence microscopy $(40 \times$, bar $=100 \mu \mathrm{m}) . A D$, adults. In the panel labeled as AD $63 \times$, the localization of different lipid droplets is illustrated, showing their intense concentration in the interstitium. Within seminiferous tubules, two populations of lipid droplets are evident: a smaller one typically facing the tubular lumen (arrowheads) and a less numerous, larger, and more disperse type, located close to the basal membrane (arrows) of tubules. The lowest panel on the left corner shows the concentration of TAGs in the mouse PS, RS, and RB employed in this study. TAGs were quantified by their fatty acids and their concentration is expressed on the basis of a fixed amount of lipid phosphorus (P).

to define FABP3 functions in different tissues (Schachtrup et al. 2008, Shioda et al. 2010), no one has yet informed of the effects of such gene change on testicular function.

Fabp5 expression was here detected as significant in Sertoli cells. The developmentally associated decrease in its expression in whole testis may be ascribed to the relatively small proportion of these cells with respect to germ cells in the adult and with the almost negligible expression of this gene in germ cells (Figs 1 and 2). FABP5 was previously observed in bovine Sertoli cells and retinal Müller cells, both having in common that they are supportive of other cell types in their respective tissues (Kingma et al. 1998). A function of Sertoli cells is to remove, by means of phagocytosis, apoptotic bodies evolving from supernumerary spermatocytes and $\mathrm{RB}$ released from condensing spermatids. Once these lipid-containing bodies have been incorporated, and their content hydrolyzed in lysosomes, a variety of fatty acids - from saturates to long-chain and verylong-chain PUFA - need to be transported to subcellular organelles such as the endoplasmic reticulum, mitochondria, or peroxisomes to be used in biosynthetic and/or catabolic pathways. The high level of Fabp5 expression that we found in mouse Sertoli cells points to a highly active intracellular fatty acid traffic among organelles. The fact that this protein has low ligand specificity, as it binds fatty acids with widely differing levels of unsaturation with relatively high affinities (Kingma et al. 1998), agrees with this possibility.

The mRNA of Fabp7, mainly detected in brain, was included in our study because this member of the Fabp family was occasionally detected in the zebrafish (Danio rerio) testis (Liu et al. 2003) and because it is involved in the transport of the 22-carbon PUFA-like docosahexaenoic acid (Balendiran et al. 2000, Mita et al. 2010). This fatty acid, like docosapentaenoic acid, abounds in mouse testis lipids (Furland et al. 2003), where it is highly concentrated in membrane GPLs and TAGs, of germ cells and RB (present results). Further work is required to clarify the apparent discrepancy between the faint expression of Fabp7 (present results) and the abundance of 22-carbon PUFA in mouse testis lipids.

FABP9 is the best characterized member of the FABP family in the testis. The fact that both increase in ascending order from PS to RS, from these cells to ES, and from these cells to RB explains why Fabp9 mRNA and protein accumulate during postnatal maturation in the testis. The presence of FABP9 protein at the latest stages of spermatid differentiation agrees with previous observations using immunohistochemistry in testis sections (Korley et al. 1997, Selvaraj et al. 2010). Our results detecting this protein in isolated $\mathrm{ES}$ and $\mathrm{RB}$ agree with the presence of this protein in mouse spermatozoa (Korley et al. 1997). Interestingly, FABP9 is one of the proteins whose phosphorylation state increases during in vitro sperm capacitation (Platt et al. 2009).

The Sertoli cells used in this study, in addition to being obtained at a relatively early developmental stage (P18), were cultured for 2 weeks in order to exclude remainders of germ cells as possible sources of this gene. Although these conditions may be considered with caution, as the transcripts could have been degraded during that time, the same conditions served to demonstrate high and specific Fabp5 (and other mRNAs) expression by these cells. Our observation that FABP9, both at the mRNA and protein levels, was undetectable in Sertoli cells supports the notion that it is not endogenous to these cells. The fact that it is detectable in Sertoli cells after a massive apoptosis of spermatocytes is induced is probably a result of Sertoli cell phagocytic activity (Kido \& Namiki 2000). 


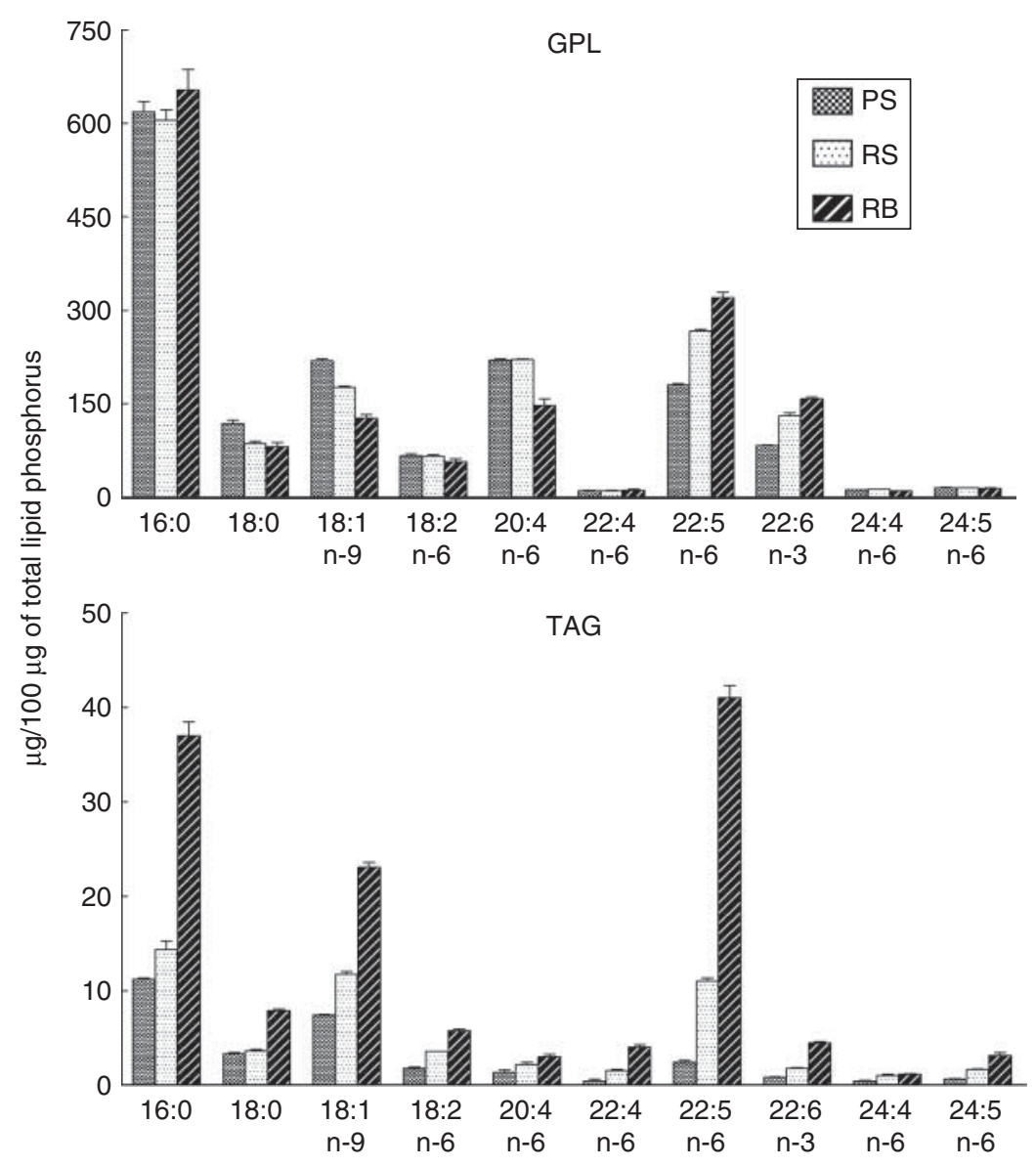

Figure 6 Differential amounts of main fatty acids from GPLs and TAGs in the mouse germ cells and residual bodies analyzed in this study.
It was intriguing that during the last stages of germ cell differentiation, both Fabp9 and Fabp12 expression increased at the mRNA level but apparently not both at the protein level. Problems with the immunodetection were not likely, as both proteins were expressed at similar rates during meiosis (PS) and up to early steps of spermiogenesis (RS), the main difference being established in ES and RB. This discrepancy could bear a relationship with post-transcriptional regulation via RNA, whereby the latter is silenced concomitantly with mouse germ cell differentiation (Gonzalez-Gonzalez et al. 2008), possibly affecting the expression of each of these proteins to different extents.

In general, FABPs participate mainly in the uptake and intracellular traffic of fatty acids and acyl-CoAs prior to their utilization in cells (Liou \& Storch 2001). Because Fabp9 not only abounded but increased in cells in the late stages of spermatogenesis as well as RB, we suggest that the protein could contribute to the intracellular transport of those fatty acids that are required for the synthesis of the membrane sphingolipids and GPLs that will exit from the testis together with the mature gametes. The protein could also contribute to the conservation of fatty acids within the testis by transporting the surplus of fatty acids discarded during spermatid volume reduction to the subcellular site of TAG synthesis. This possibility is suggested by the fact, confirmed here, that these neutral glycerides concentrate a lot of the most metabolically costly PUFAs (e.g. 22:5n-6 and 22:6n-3). Further studies are still needed to establish the nature of the hydrophobic ligands of FABP9 and clarify its role in spermatogenesis. Intriguingly, mice lacking Fabp9 are viable and fertile, and the total lipid fatty acid profile of their spermatozoa is not apparently affected (Selvaraj et al. 2010). It is then possible that the deficiency in FABP9 could be compensated by overexpression of Fabp12 in the germ cells of Fabp9-null mice, thereby enabling spermatogenesis to continue. Quantitative analysis of expression of other FABPs, particularly of Fabp12, in Fabp9-null mice would be important to confirm the suggested co-regulation of FABPs in the testis.

Our finding that the testis contains transcripts encoding for both Dgat1 and Dgat2 and that these genes differ in their degree of upregulation with testicular development and in their distribution among cells suggest their involvement in TAG biosynthesis as part of testicular lipid homeostasis. TAGs are the main neutral lipids present in adult rodent seminiferous tubules (Furland et al. 2003). The fact that Dgat2 was 
increasingly expressed from spermatocytes to spermatids and from these cells to RB is in agreement with the concurrently progressive increase in TAG levels, we observed in rodent testis in this sequence (Oresti et al. 2010 and present results).

The biosynthesis of TAG was proposed to function as one of the strategies used by developing spermatids while reducing their volume to become thinner and longer before being released as spermatozoa into the tubular lumen (Oresti et al. 2010). Intracellular organelles such as Golgi membranes, ribosomes, or peroxisomes, which have important biosynthetic functions in germ cells during earlier stages, are no longer required in gametes. In the last stages of spermiogenesis, all surplus material in each spermatid including lipids is tightly condensed and enclosed in a subacrosomal cytoplasmic lobe, which is then released in compact form as an RB (Russell et al. 1990). The volume occupied by membrane GPLs may be efficiently reduced by converting them partly into diacylglycerols and partly into free fatty acids, then combining these products into TAGs, and confining such TAGs into lipid droplets.

In addition to diglycerides, DGAT2 requires fatty acids or acyl-CoAs to be carried to the site of TAG synthesis, a function that could be played by FABP9 - or alternatively by FABP12. Subsequently, TAGs need to be transported to, and stored in, lipid droplets. The increasing amounts of these subcellular inclusions with germ cell differentiation agrees with the finding that Plin2 mRNA level was higher in spermatids and RB than in spermatocytes. The differentiation-related increase in the amounts of TAG and lipid droplets, concomitant with the increase in Fabp9, Dgat2, and Plin2 expression as spermatogenesis progresses suggests a functional connection between the products of these transcripts. The finding of a high concentration of these three mRNAs in RB is intriguing, but reasonable if one considers that they have terminated their function related to protein synthesis in spermatids. In fact, these mRNAs are a minor part of the total RNA that RB were shown to contain (Shin et al. 2007). Although the ultimate biochemical fate of the RNA molecules confined in these particles remains to be established, the possibility that some of their hydrolysis products or related metabolites could serve regulatory functions relevant to spermatogenesis in Sertoli cells cannot be excluded.

\section{Supplementary data}

This is linked to the online version of the paper at http://dx. doi.org/10.1530/REP-13-0199.

\section{Declaration of interest}

The authors declare that there is no conflict of interest that could be perceived as prejudicing the impartiality of the research reported.

\section{Funding}

This study is supported by grants from The European Chemical Industry, Council-Long-range Research Initiative (CEFIC-LRi), MEDDTL (11-MRES-PNRPE-9-CVS-072) (France), and CSIC (PIE 201020E016) (Spain). G M Oresti was a visiting postdoctoral researcher from SPU, Ministerio de Educación (Argentina).

\section{References}

Albert DH, Ascoli M, Puett D \& Coniglio JG 1980 Lipid composition and gonadotropin-mediated lipid metabolism of the M5480 murine Leydig cell tumor. Journal of Lipid Research 21 862-867.

Balendiran GK, Schnutgen F, Scapin G, Borchers T, Xhong N, Lim K, Godbout R, Spener F \& Sacchettini JC 2000 Crystal structure and thermodynamic analysis of human brain fatty acid-binding protein. Journal of Biological Chemistry 275 27045-27054. (doi:10.1074/jbc. M003001200)

Beckman JK, Gray ME \& Coniglio JG 1978 The lipid composition of isolated rat spermatids and spermatocytes. Biochimica et Biophysica Acta $\mathbf{5 3 0}$ 367-374. (doi:10.1016/0005-2760(78)90156-X)

Bellve AR, Cavicchia JC, Millette CF, O'Brien DA, Bhatnagar YM \& Dym M 1977 Spermatogenic cells of the prepubertal mouse. Isolation and morphological characterization. Journal of Cell Biology 74 68-85. (doi:10.1083/jcb.74.1.68)

Bligh EG \& Dyer WJ 1959 A rapid method of total lipid extraction and purification. Canadian Journal of Biochemistry and Physiology 37 911-917. (doi:10.1139/059-099)

Brasaemle DL, Barber T, Wolins NE, Serrero G, Blanchette-Mackie EJ \& Londos C 1997 Adipose differentiation-related protein is an ubiquitously expressed lipid storage droplet-associated protein. Journal of Lipid Research 38 2249-2263.

Cases S, Smith SJ, Zheng YW, Myers HM, Lear SR, Sande E, Novak S, Collins C, Welch CB, Lusis AJ et al. 1998 Identification of a gene encoding an acyl CoA:diacylglycerol acyltransferase, a key enzyme in triacylglycerol synthesis. PNAS 95 13018-13023. (doi:10.1073/pnas.95. 22.13018)

Cases S, Stone SJ, Zhou P, Yen E, Tow B, Lardizabal KD, Voelker T \& Farese RV Jr 2001 Cloning of DGAT2, a second mammalian diacylglycerol acyltransferase, and related family members. Journal of Biological Chemistry 276 38870-38876. (doi:10.1074/jbc.M106219200)

Castillo AF, Maciel FC, Castilla R, Duarte A, Maloberti P, Paz C \& Podesta EJ 2006 cAMP increases mitochondrial cholesterol transport through the induction of arachidonic acid release inside this organelle in Leydig cells. FEBS Journal 273 5011-5021. (doi:10.1111/j.1742-4658. 2006.05496.x)

Chauvigne F, Plummer S, Lesne L, Cravedi JP, Dejucq-Rainsford N, Fostier A \& Jegou B 2011 Mono-(2-ethylhexyl) phthalate directly alters the expression of Leydig cell genes and CYP17 lyase activity in cultured rat fetal testis. PLoS ONE 6 e27172. (doi:10.1371/journal.pone.0027172)

Chmurzynska A 2006 The multigene family of fatty acid-binding proteins (FABPs): function, structure and polymorphism. Journal of Applied Genetics 47 39-48. (doi:10.1007/BF03194597)

Furland NE, Maldonado EN \& Aveldano MI 2003 Very long chain PUFA in murine testicular triglycerides and cholesterol esters. Lipids 38 73-80. (doi:10.1007/s11745-003-1033-9)

Furuhashi M \& Hotamisligil GS 2008 Fatty acid-binding proteins: role in metabolic diseases and potential as drug targets. Nature Reviews. Drug Discovery 7 489-503. (doi:10.1038/nrd2589)

Gonzalez-Gonzalez E, Lopez-Casas PP \& del Mazo J 2008 The expression patterns of genes involved in the RNAi pathways are tissuedependent and differ in the germ and somatic cells of mouse testis. Biochimica et Biophysica Acta 1779 306-311. (doi:10.1016/j.bbagrm. 2008.01.007)

Grogan WM \& Huth EG 1983 Biosynthesis of long-chain polyenoic acids from arachidonic acid in cultures of enriched spermatocytes and spermatids from mouse testis. Lipids 18 275-284. (doi:10.1007/ BF02534702) 
Haunerland NH \& Spener F 2004 Fatty acid-binding proteins - insights from genetic manipulations. Progress in Lipid Research 43 328-349. (doi:10.1016/j.plipres.2004.05.001)

Heid HW, Moll R, Schwetlick I, Rackwitz HR \& Keenan TW 1998 Adipophilin is a specific marker of lipid accumulation in diverse cell types and diseases. Cell and Tissue Research 294 309-321. (doi:10.1007/ s004410051181)

Heuckeroth RO, Birkenmeier EH, Levin MS \& Gordon JI 1987 Analysis of the tissue-specific expression, developmental regulation, and linkage relationships of a rodent gene encoding heart fatty acid binding protein. Journal of Biological Chemistry 262 9709-9717.

Karl AF \& Griswold MD 1990 Sertoli cells of the testis: preparation of cell cultures and effects of retinoids. Methods in Enzymology 190 71-75. (doi:10.0076-6879(90)90010-X)

Kerr JB \& De Kretser DM 1974 Proceedings: The role of the Sertoli cell in phagocytosis of the residual bodies of spermatids. Journal of Reproduction and Fertility 36 439-440. (doi:10.1530/jrf.0.0360439)

Kido T \& Namiki H 2000 Expression of testicular fatty acid-binding protein PERF 15 during germ cell apoptosis. Development, Growth \& Differentiation 42 359-366. (doi:10.1046/j.1440-169x.2000.00520.x)

Kido T, Arata S, Suzuki R, Hosono T, Nakanishi Y, Miyazaki J, Saito I, Kuroki T \& Shioda S 2005 The testicular fatty acid binding protein PERF15 regulates the fate of germ cells in PERF15 transgenic mice. Development, Growth \& Differentiation 47 15-24. (doi:10.1111/j.1440169x.2004.00775.x)

Kimmel AR, Brasaemle DL, McAndrews-Hill M, Sztalryd C \& Londos C 2010 Adoption of PERILIPIN as a unifying nomenclature for the mammalian PAT-family of intracellular lipid storage droplet proteins. Journal of Lipid Research 51 468-471. (doi:10.1194/jlr.R000034)

Kingma PB, Bok D \& Ong DE 1998 Bovine epidermal fatty acid-binding protein: determination of ligand specificity and cellular localization in retina and testis. Biochemistry 37 3250-3257. (doi:10.1021/bi972520l)

Korley R, Pouresmaeili F \& Oko R 1997 Analysis of the protein composition of the mouse sperm perinuclear theca and characterization of its major protein constituent. Biology of Reproduction 57 1426-1432. (doi:10.1095/ biolreprod57.6.1426)

Lehner R \& Kuksis A 1996 Biosynthesis of triacylglycerols. Progress in Lipid Research 35 169-201. (doi:10.1016/0163-7827(96)00005-7)

Liou HL \& Storch J 2001 Role of surface lysine residues of adipocyte fatty acid-binding protein in fatty acid transfer to phospholipid vesicles. Biochemistry 40 6475-6485. (doi:10.1021/bi0101042)

Liu RZ, Denovan-Wright EM \& Wright JM 2003 Structure, mRNA expression and linkage mapping of the brain-type fatty acid-binding protein gene (FABP7) from zebrafish (Danio rerio). European Journal of Biochemistry 270 715-725. (doi:10.1046/j.1432-1033.2003.03432.x)

Liu RZ, Li X \& Godbout R 2008 A novel fatty acid-binding protein (FABP) gene resulting from tandem gene duplication in mammals: transcription in rat retina and testis. Genomics 92 436-445. (doi:10.1016/j.ygeno. 2008.08.003)

Livak KJ \& Schmittgen TD 2001 Analysis of relative gene expression data using real-time quantitative PCR and the 2 (-Delta Delta $C(\mathrm{~T}))$ method. Methods 25 402-408. (doi:10.1006/meth.2001.1262)

McIntosh AL, Senthivinayagam S, Moon KC, Gupta S, Lwande IS, Murphy CC, Storey SM \& Atshaves BP 2012 Direct interaction of Plin2 with lipids on the surface of lipid droplets: a live cell FRET analysis. American Journal of Physiology. Cell Physiology 303 C728-C742. (doi:10.1152/ajpcell.00448.2011)

Mita R, Beaulieu MJ, Field C \& Godbout R 2010 Brain fatty acid-binding protein and $\omega-3 / \omega-6$ fatty acids: mechanistic insight into malignant glioma cell migration. Journal of Biological Chemistry 285 37005-37015. (doi:10.1074/jbc.M110.170076)

Murphy EJ, Owada Y, Kitanaka N, Kondo H \& Glatz JF 2005 Brain arachidonic acid incorporation is decreased in heart fatty acid binding protein gene-ablated mice. Biochemistry 44 6350-6360. (doi:10.1021/ bi047292r)
Ockner RK, Manning JA \& Kane JP 1982 Fatty acid binding protein. Isolation from rat liver, characterization, and immunochemical quantification. Journal of Biological Chemistry 257 7872-7878.

Oresti GM, Reyes JG, Luquez JM, Osses N, Furland NE \& Aveldaño MI 2010 Differentiation-related changes in lipid classes with long-chain and very long-chain polyenoic fatty acids in rat spermatogenic cells. Journal of Lipid Research 51 2909-2921. (doi:10.1194/jlr.M006429)

O'Shaughnessy PJ, Willerton L \& Baker PJ 2002 Changes in Leydig cell gene expression during development in the mouse. Biology of Reproduction 66 966-975. (doi:10.1095/biolreprod66.4.966)

Paz M, Morin M \& del Mazo J 2006 Proteome profile changes during mouse testis development. Comparative Biochemistry and Physiology. Part D, Genomics \& Proteomics 1 404-415. (doi:10.1016/j.cbd.2006.10.002)

Platt MD, Salicioni AM, Hunt DF \& Visconti PE 2009 Use of differential isotopic labeling and mass spectrometry to analyze capacitationassociated changes in the phosphorylation status of mouse sperm proteins. Journal of Proteome Research 8 1431-1440. (doi:10.1021/pr800796j)

Romrell LJ, Bellve AR \& Fawcett DW 1976 Separation of mouse spermatogenic cells by sedimentation velocity. A morphological characterization. Developmental Biology 49 119-131. (doi:10.1016/ 0012-1606(76)90262-1)

Russell LD, Ettlin RA, Sinha Hikim AP \& Clegg ED 1990 Mammalian spermatogenesis. In Histological and Histopathological Evaluation of the Testis. edn 1, pp 1-38. St Louis: Cache River Press.

Schachtrup C, Malcharek S, Haitsma JJ, Lachmann B, Owada Y, Binas B, Kondo H, Rustow B, Galla HJ \& Spener F 2008 Activation of PPAR $\gamma$ reverses a defect of surfactant synthesis in mice lacking two types of fatty acid binding protein. Biochimica et Biophysica Acta 1781 314-320. (doi:10.1016/j.bbalip.2008.04.010)

Selvaraj V, Asano A, Page JL, Nelson JL, Kothapalli KS, Foster JA, Brenna JT, Weiss RS \& Travis AJ 2010 Mice lacking FABP9/PERF15 develop sperm head abnormalities but are fertile. Developmental Biology 348 177-189. (doi:10.1016/j.ydbio.2010.09.019)

Shin M, Larsson LI \& Fujiwara K 2007 Polyamines in spermatocytes and residual bodies of rat testis. Histochemistry and Cell Biology 127 649-655. (doi:10.1007/s00418-007-0285-7)

Shioda N, Yamamoto Y, Watanabe M, Binas B, Owada Y \& Fukunaga K 2010 Heart-type fatty acid binding protein regulates dopamine D2 receptor function in mouse brain. Journal of Neuroscience $\mathbf{3 0}$ 3146-3155. (doi:10.1523/JNEUROSCI.4140-09.2010)

Spener F, Unterberg C, Borchers T \& Grosse R 1990 Characteristics of fatty acid-binding proteins and their relation to mammary-derived growth inhibitor. Molecular and Cellular Biochemistry 98 57-68. (doi:10.1007/ BF00231368)

Watanabe M, Ono T \& Kondo H 1991 Immunohistochemical studies on the localisation and ontogeny of heart fatty acid binding protein in the rat. Journal of Anatomy 174 81-95.

Yamamoto T, Yamamoto A, Watanabe M, Matsuo T, Yamazaki N, Kataoka M, Terada H \& Shinohara Y 2009 Classification of FABP isoforms and tissues based on quantitative evaluation of transcript levels of these isoforms in various rat tissues. Biotechnology Letters 31 1695-1701. (doi:10.1007/s10529-009-0065-7)

Zanetti SR, de Los Angeles MM, Rensetti DE, Fornes MW \& Aveldaño MI 2010 Ceramides with 2-hydroxylated, very long-chain polyenoic fatty acids in rodents: from testis to fertilization-competent spermatozoa. Biochimie 92 1778-1786. (doi:10.1016/j.biochi.2010.08.012)

Zschiesche W, Kleine AH, Spitzer E, Veerkamp JH \& Glatz JF 1995 Histochemical localization of heart-type fatty-acid binding protein in human and murine tissues. Histochemistry and Cell Biology 103 147-156. (doi:10.1007/BF01454012)

Received 9 May 2013

First decision 8 July 2013

Accepted 20 August 2013 